\title{
Finite Element Modeling and Simulation of Car Crash
}

\author{
Andrew Hickey, Shaoping Xiao \\ 3131 Seamans Center, Department of Mechanical and Industrial Engineering \\ The University of Iowa, Iowa City, Iowa 52242
}

\begin{abstract}
In this paper, quasi-static simulations were conducted to simulate car crash via finite element method. A 2002 Ford explorer was modeled in the 3D modeling software CREO and then imported into ANSYS for mesh generation and FEM analysis. Various incoming speeds were considered when the car was modeled to crash into a wall. It was observed that when the car with an incoming speed of 100mph, the car was totaled. The maximum deformation in numerical simulation agreed with the one from a real life testing.
\end{abstract}

Keywords: car crash, finite element, ANSYS, deformation

\section{INTRODUCTION}

Safety is one of the design considerations in automobile community. Therefore, crash test is an important step to validate the novel car design. However, high cost in experimental testing limits the number of crash tests, and adequate data might not be obtained consequently. Alternatively, numerical modeling and simulation have been widely used to study car crash in addition to experimental testing. As a powerful numerical tool, finite element method (FEM) [1] plays an vital role in crash test simulations.

Lin and co-workers [2] developed a numerical model for the computer simulation of car crash analysis. They analyzed two crash situations: a high-speed vehicle crashing into a wall and a high speed test vehicle crashing into a static vehicle. Their research objective was to identify the sources of harm to driver and passengers when car crashes occur. On the other hand, the major concern in design of bumper is its potentiality to bear impact loads. Numerical simulations are also normally used to assure a bumper design to meet the safety requirements. Kankariya and Sayyad [3] employed an explicit FEM to investigate stress and effective plastic strain of bumper at impact. They recommended modifications in bumper design to improve its impact performance based on the simulation results. In addition, Sun and co-workers [4] carried out CAE simulation with high performance computing techniques. Their simulation of a FEM car model was in a distributed computation environment with the development of coding-and-programming of DAYN3D. There were almost ten numerical automobile models studied in their research, which provided various computational examples and a user handbook.

In this paper, we simulated a car crashing into the wall virtually to understand the devastating outcome that car crashes can have on automobiles. To simplify the study, only the car frame was considered in our studies. The car model was generated in the $3 \mathrm{~d}$ modeling software CREO and then imported to the FEM analysis software ANSYS for mesh generation and FEM analysis. We adopted quasi-static simulation tool in ANSYS, and employed the conservation of linear momentum to calculate the time-averaged force acting on the car during the impact. Therefore, the wall was not modeled in our studies. Various incoming velocities were considered, and the car deformation was compared to the one from a real life testing.

The outline of this paper is described as below. After introduction, the methodologies, including car model generation and FEM analysis, are given in Section 2. Results are discussed in Section 3 followed by the conclusions.

\section{Methodologies}

The design of the car frame was generated in the 3D modeling software CREO, where the car was created as a life size model to accurately examine the effects of a car crash. The car studied here was a 
2002 Ford Explorer as shown in Figure 1. The dimensions of the car were researched online on the Ford website and translated into the design in CREO. The overall dimensions of a 2002 explorer are approximately 71"x190"x 71 " (W x L x H). Figures 2 depicts the model of the car that was generated. As mentioned before, only the frame of the explorer was generated in order to analyze how the frame structure deforms during the impact of a crash.

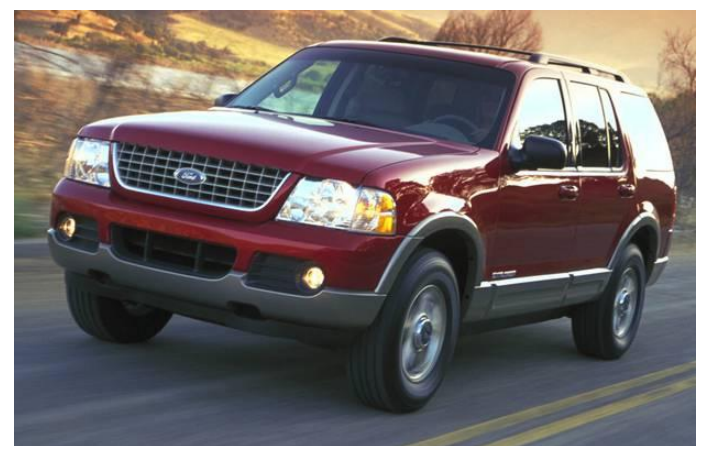

Figure1. Isometric view of the 2002 Ford Explorer in real life

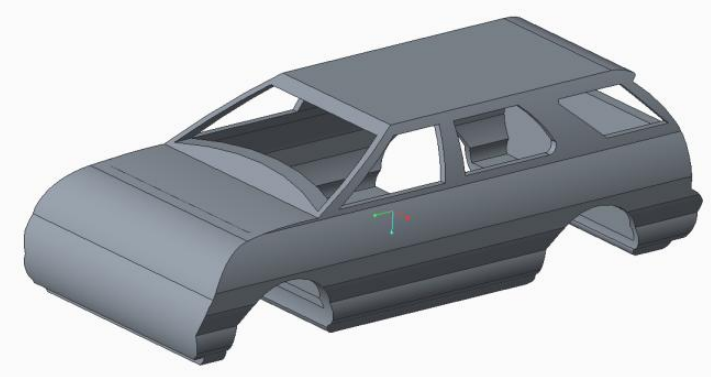

Figure2. Isometric view of the 2002 Ford Explorer in CREO

The geometry was exported as an IGS file from CREO and then imported into ANSYS for mesh generation and FEM analysis. Upon importing the geometry, the material was set to aluminum alloy for the car body. The mass of a Ford Explorer is approximately $2458 \mathrm{~kg}$, based on the information on the Ford website. When the CREO model was transferred into ANSYS, the mass was measured to be $2327.84 \mathrm{~kg}$, which is accurate to the real life model. A tetrahedral mesh was generated on the car as shown in Figure 3. At points of finer detail, there are more nodes and elements are smaller because the geometry is more complex and thus better approximations must be made. The number of elements and nodes in the FEM model of the car are 160293 and 35504 respectively.

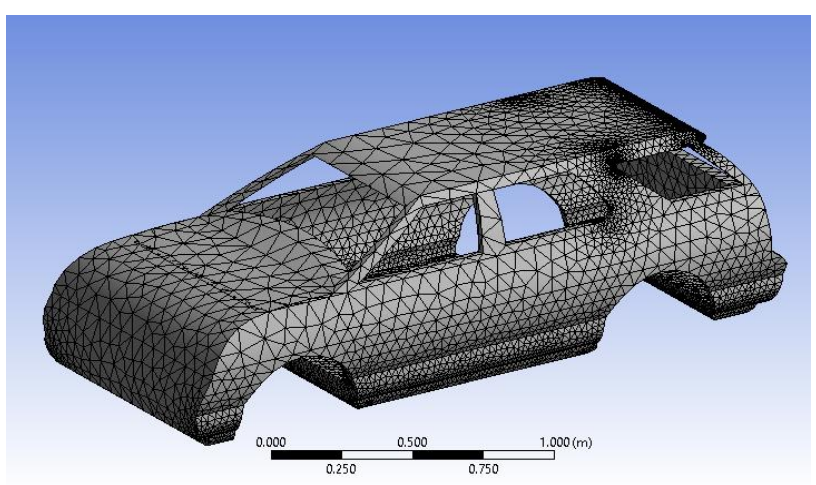

Figure3. Mesh generation for the 2002 Ford Explorer in ANSYS

In this paper, quasi-static simulations were conducted, and the car was subjected to loads at specific nodes in the above FEM model. Based on the conservation of linear momentum, Equation (1) below was used to solve for the time averaged force $F$ that would be distributed at each node on the front of the car.

$F^{*} \Delta t=-m V_{l}$

where $\Delta t=1 \mathrm{~s}$ is the duration of impact, $m$ is the car mass, and $V_{l}$ is the incoming speed of car before impact. 
A fine mesh was placed on the front of the car to accurately depict the event of a crash, where a wall would hit the whole front of the car rather than at different points on the fender, which is what a coarse mesh would provide. This of course is assuming that the car hits the wall directly head on at a perpendicular angle to the face of the wall. It shall be noted that the total force was calculated in Equation (1) at first. Then the nodal forces, applied at the nodes as shown in Figure 4, need to be determined via the FEM approximation. A total of 162 nodes had multiple different forces applied to it. All nodal forces in Figure 4 are labeled in pink.

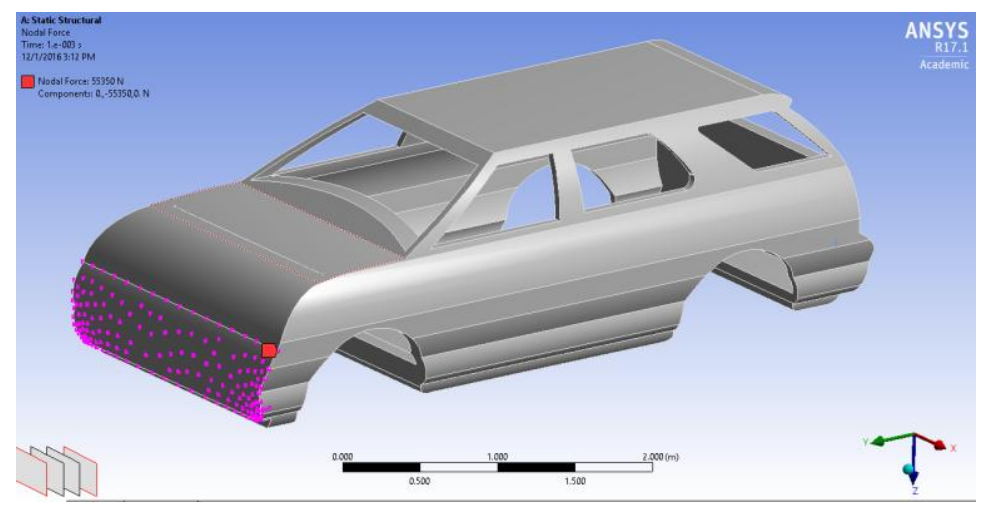

Figure4. Applied nodal forces on contact points of car

\section{RESULTS AND DISCUSSIONS}

Various incoming speeds, from $20 \mathrm{mph}$ to $100 \mathrm{mph}$ in increments of $20 \mathrm{mph}$, were considered here as the speeds of car before impact. Table 1 lists the total forces calculated via Equation (1), and the maximum deformations after impact. The deformed configurations of car after impact are shown in Figures 5 - 7.

As seen in Figures 5-7, the car deformed on different magnitudes for each test speed. The crash at $20 \mathrm{mph}$ leaves the car with deformation, but nothing that would cause any harm to the driver or passengers. Whereas, a crash at $100 \mathrm{mph}$ will leave a car totaled and most likely result in fatality as seen in Figure 7. In the car crash simulation at $100 \mathrm{mph}$ the maximum deformation was calculated to be 73.44in, which is approximately 6ft. Discovery channel's Myth Busters performed a real impact test on a car, shown in Figure 8, similar to the simulation done in this paper. In Myth busters car testing, a car travelled at $100 \mathrm{mph}$ into a wall. These result, at impact, show that the car nearly deformed half of its length. The total length of the explorer is 190", therefore with a deformation of 73 ", it can be concluded that the our simulations are validated.

Table1. Force magnitudes and maximum deformations at various car speeds.

\begin{tabular}{|c|c|c|c|}
\hline Car Speed (mph) & Force (N) & Max Deformation (m) & Max Deformation (in) \\
\hline 20 & 20812.75187 & $2.78 \mathrm{E}-01$ & 10.9271435 \\
\hline 40 & 41625.50374 & 0.50729 & 19.9720073 \\
\hline 60 & 62438.25562 & 0.80594 & 31.7298578 \\
\hline 80 & 83251.00749 & 1.4262 & 56.149494 \\
\hline 100 & 104063.7594 & 1.8656 & 73.448672 \\
\hline
\end{tabular}

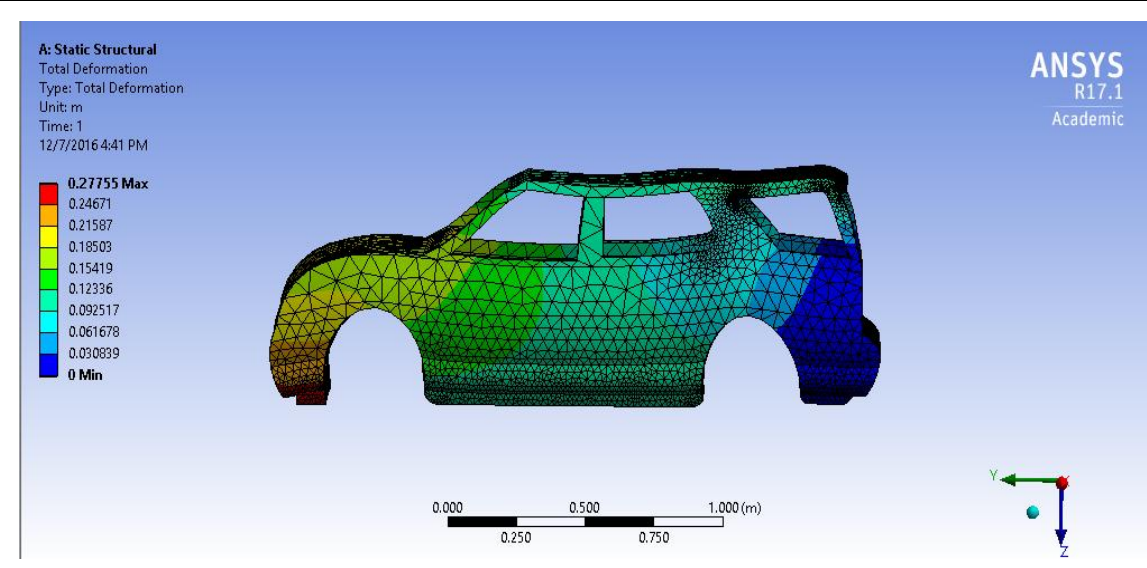

Figure5. Total deformation of car at $20 \mathrm{mph}$ 


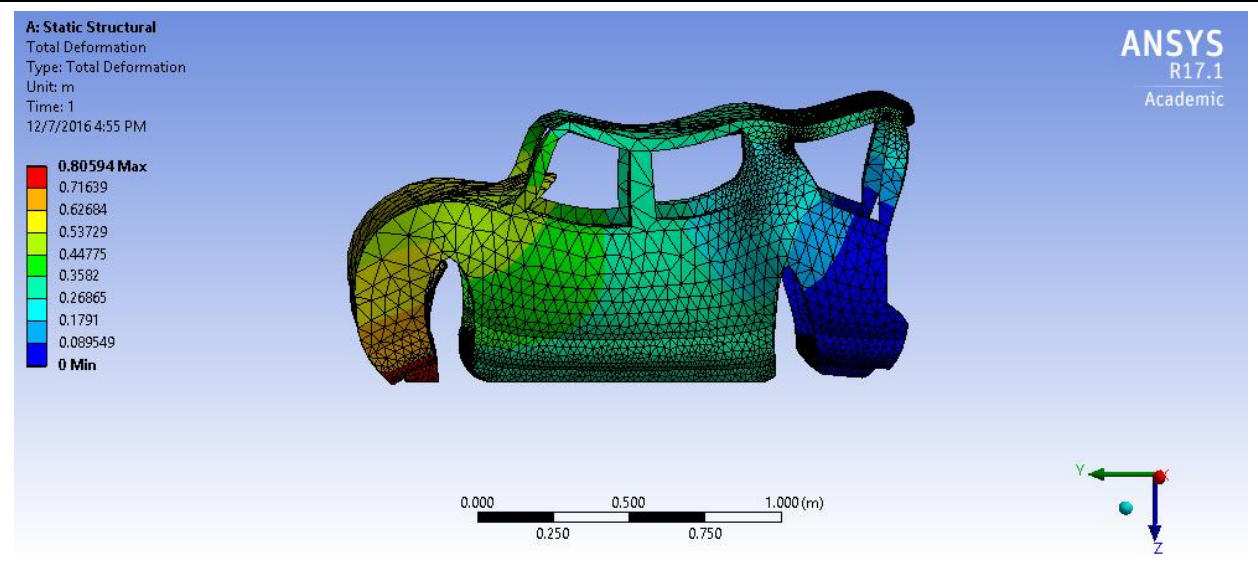

Figure6. Total deformation of car at $60 \mathrm{mph}$

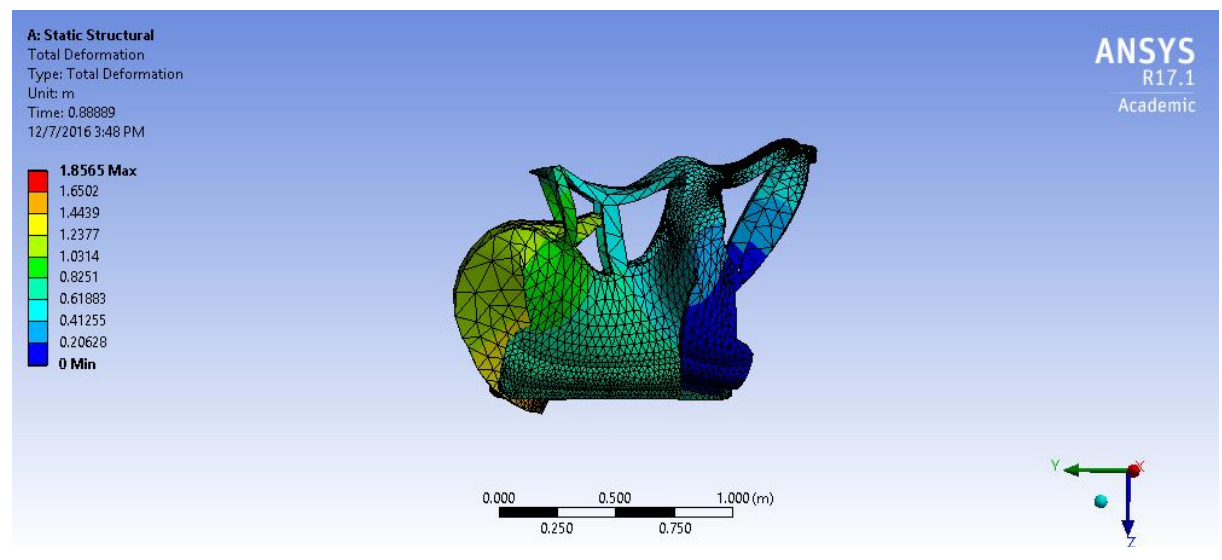

Figure7. Total deformation of car at 100mph

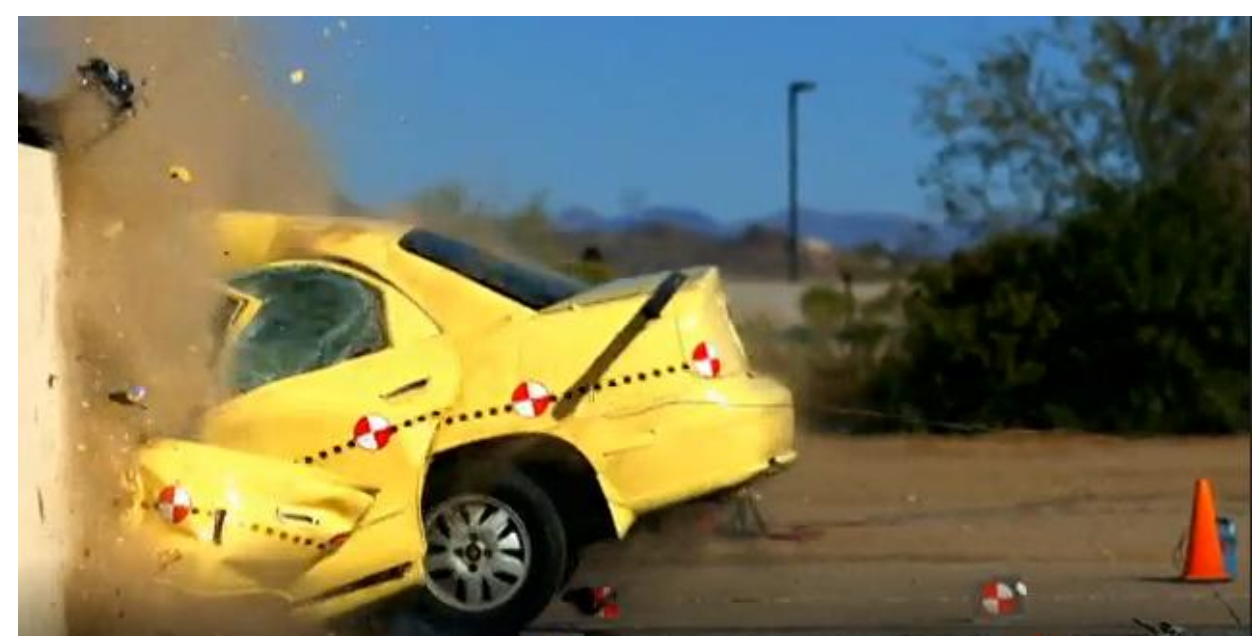

Figure8. Deformation of car at 100mph at impact as tested by Myth Busters

However, when looking at the visual deformation between the simulation and real life test, there are some discrepancies. One noticeable discrepancy is the difference in car size and deformation. An SUV, like the Ford Explorer, would deform much less than the smaller car seen in the MythBusters test. A plausible reason for this phenomenon is because in the simulation, only the frame of the car was used, whereas the real test car has more mass to it from the interior, engine, and chassis. Another explanation for the difference in deformation between the simulation and real test is the amount of moving parts on the real life test. An actual car is made of hundreds of parts that are held together with bolts, screws and fasteners. In the simulation, the model created was generated as one body to make the meshing and solution solving easier for the computer. With more moving parts it would take a long time for the computer to solve the values that were desired to examine the car. More moving parts create more destruction and deformations in different directions. Figure 8 shows deformations in all directions, whereas the simulations shown in Figures $5-7$ showed most of the deformation in the $y$ direction, which is the direction of motion. 


\section{Conclusions}

The numerical modeling and simulation of car crash have been carried on in automobile companies for years. Finite element analysis can generate realistic results that help scientists and engineers understand the way that cars are effected by different crash scenarios. Instead of running real life situations, it is much more cost effective to simulate car crash using a commercial software such as ANSYS. Although the simulation conducted here is not as in depth as one done by an actual car company, the results displayed in this paper show what these simulations have to offer to not only the car community, but also the engineering community. With the development of commercial software, it is imperative that engineers continue to use computer to simulate real life scenarios, estimate the outcomes, and develop new technology to help save lives in the events of car crashes.

\section{REFERENCE}

[1] T. Belytschko, W.K. Liu and B. Moran, Nonlinear Finite Elements for Continua and Structures, 1st ed. U.S.: Wiley, New York, 2001.

[2] Lin C. S., Chou K. D. and Yu C. C., Numerical simulation of vehicle crashes, Appl. Mech. Mat. 590, 135 (2014).

[3] Kankariya N. and Sayyad F. B., Numerical simulation of bumper impact analysis and to improve design for crash worthiness, Int. J. Engrg. Sci. 4(5), 58 (2015).

[4] Sun T., Liu T., Shen I F. and Ma Y. S., Numerical simulation of car rash analysis based on distributed computational environment, Conference Proceeding, 5th Int. Conference on Algorithms and Architectures for Parallel Processing, Pp 334-337, (2002).

\section{AUTHORS' BIOGRAPHY}

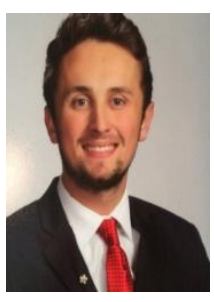

Andrew Hickey, is a Mechanical Engineering student at the University of Iowa with a focus in product design and development. He is graduating in May of 2017 with a Bachelors degree in Mechanical Engineering.

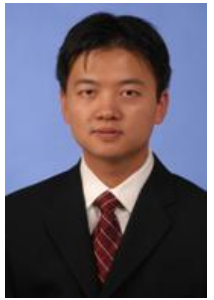

Shaoping Xiao, is an associate professor in the Department of Mechanical and Industrial Engineering at The University of Iowa. He was graduated by Northwestern University with a $\mathrm{PhD}$ degree in mechanical engineering in 2003 before joining The University of Iowa. His group's research interests include finite element method, meshfree particle methods, multiscale modeling and simulation. 